\title{
Research Paper: Diagnostic Challenges and Prospects Associated With Zoonotic Tuberculosis of Central Nervous System
}

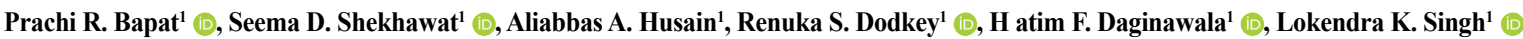
Rajpal S. Kashyap ${ }^{1 *}$ Challenges and Prospects Associated With Zoonotic Tuberculosis of Central Nervous System. Basic and Clinical Neuroscience, 11(5), 619-630. http://dx.doi.org/10.32598/bcn.11.5.10.803.3

doi $h$ http://dx.doi.org/10.32598/ben.11.5.10.803.3

\section{(c) (i) (\$)}

\section{Article info:}

Received: 29 Nov 2019

First Revision: 10 Dec 2019

Accepted: 11 Mar 2020

Available Online: 01 Sep 2020

Keywords:

Duplex PCR, Mycobacterium Bovis, Tuberculous meningitis, Zoonoses

\section{A B S T RA C T}

Introduction: The diagnosis of Tuberculous Meningitis (TBM) has remained a challenge due to its insidious onset and the failure of conventional diagnostic tests. The present study aimed to identify the mycobacterial pathogen in the CSF of patients with TBM and a poor prognosis.

Methods: We retrospectively recruited $224 \mathrm{TBM}$ and 34 non-TBM patients admitted to the Central India Institute of Medical Sciences, Nagpur, India, in 2014. The CSF samples of these patients were subjected to a duplex PCR assay for the species-specific identification of the causative pathogen.

Results: M. bovis and infection with M.tuberculosis were detected in 7\% (18) and 32.9\% (85) of the patients, respectively. Moreover, $14 \%$ (36) of the study samples were culture positive; however, the mycobacterial pathogens could not be differentiated to the species level.

Conclusion: The present study findings emphasized the potentially vital importance of $\mathrm{M}$. bovis identification for appropriate patient management. The obtained data also demonstrated the persistent significance of M. bovis, as a zoonotic pathogen. 


\section{Highlights}

- The study aimed at identification of M. bovis infection in CSF of TBM cases.

- Incidence of M. bovis and MTB by duplex PCR assay was 7\% \& 32.9 respectively

- The study highlights M. bovis as major zoonotic pathogen in TBM cases

- Results advocates diagnosis of M. bovis in TBM cases with poor treatment outcomes

\section{Plain Language Summary}

Tuberculosis caused by M. bovis is important yet neglected zoonotic disease of public health importance. Central nervous infection (CNS) caused by M. bovis is often associated with insidious onset due to lack of specific diagnostic tools and its intrinsic resistant to frontline TB drug pyrazinamide. Lack of specific identification \& differentiation of etiological agent in Tuberculous meningitis (TBM) infection often leads to poor treatment outcomes, high rates of neurological morbidity \& possibility of development of drug resistance. In current study we, investigated utility of inhouse designed duplex PCR assay for simultaneous diagnosis of M.tuberculosis (MTB) and M. bovis in CSF samples of TBM cases associated with poor treatment outcomes. The incidence of M. bovis by duplex PCR assay was found to be 7\% underlining its importance as important zoonotic pathogen associated with TBM infection. The study advocates utility of such molecular assay for diagnosis of M. bovis pathogen in TBM infection to improve efficacy of treatment outcome \& reduce chances of drug resistance.

\section{Introduction}

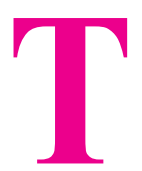

he last decade has witnessed shifting trends in Tuberculosis (TB) infection, with Extra Pulmonary Tuberculosis (EPTB) emerging as an essential entity (Jain, 2011). The TB of the Central Nervous System (CNS) is the most fatal extra-pulmonary disease that affects approximately $10 \%$ of the population globally affected with TB. The estimated mortality due to Tuberculous Meningitis (TBM) in India is 1.5 per 100000 individuals (Murthy, 2010; Kaur, et al., 2015). The diagnosis of TBM has remained a challenge to the clinicians. This is due to nonspecific clinical manifestation, which varies widely; thus, creating a major obstacle in the initiation of the treatment. Moreover, the limited sensitivity of conventional diagnostic tests (smear microscopy $\&$ culture) due to the paucibacillary nature of samples also contribute to a delayed diagnosis (Jain, 2011; Purohit \& Mustafa, 2015).

Different studies have reported the possible association between variables, such as age, the stage of the disease, clinical characteristics, mycobacterial isolation from $\mathrm{Ce}$ rebrospinal Fluid (CSF), etc., and the manifestation of TBM. The reason for poor prognosis has been ascertained to the lack of appropriate diagnostic tools for identifying the causative organism (Kaur, et al., 2015; Ahmadinejad,
Ziaee, Aghsaeifar, \& Reiskarami, 2003). Despite being a TB endemic country, limited preventive measures have been taken to improve the subsequent outcomes in patients with TBM. Despite the serious consequences following the mycobacterial infection of the CNS, our understanding of the neuro- and immunopathogenesis of cerebral mycobacterial infection is limited.

Our laboratory, for the past decade, has been working on developing immunodiagnostic and molecular tools for the clinical evaluation and appropriate management of TBM patients admitted to the Central India Institute of Medical Sciences (CIIMS); it is a tertiary healthcare facility providing medical services to the Central Indian population (Kashyap, et al., 2005; Kashyap, et al., 2006; Deshpande, et al., 2008). The majority (70\%) of those admitted here are neurology and neurosurgery patients.

In our experience, a considerable number of TBM patients do not respond to the treatment with the standard drug regimen and present neurological sequelae. The present work was thus planned as a retrospective study to assess the predictors of mortality or poor prognosis in patients diagnosed with TBM. 


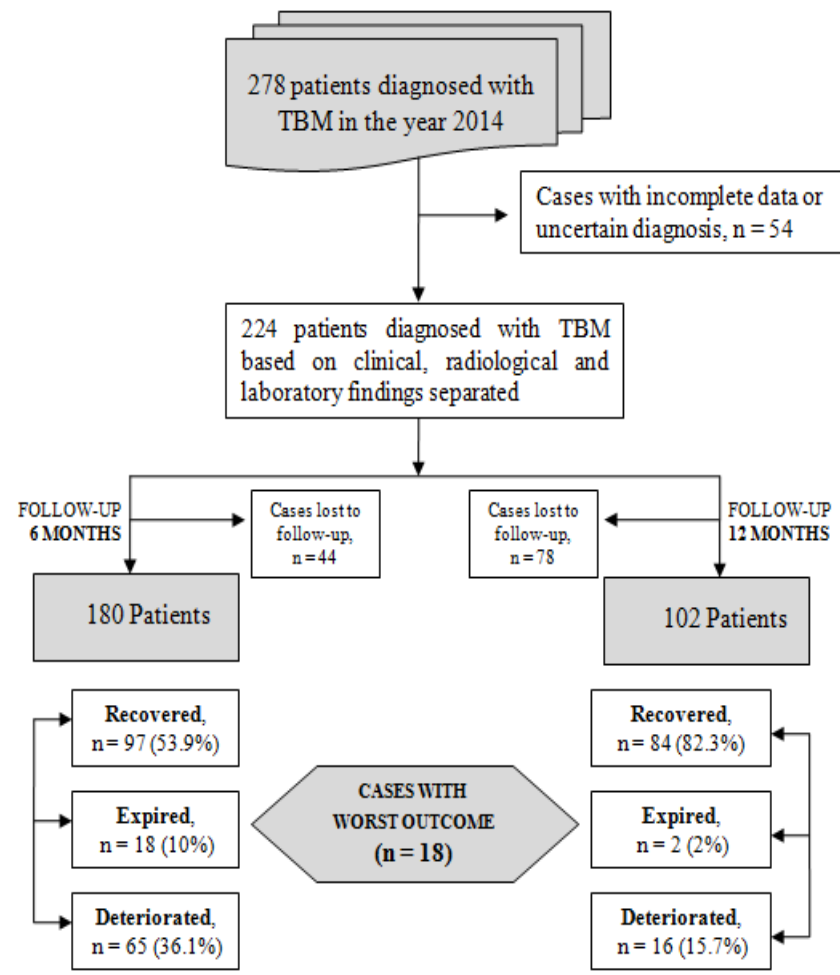

Figure 1. The schematic representation of the study design

\section{Materials and Methods}

The study was approved by the Institutional Ethics Committee of CIIMS, Nagpur City, India, and as per The Code of Ethics of the World Medical Association (the Declaration of Helsinki). Written informed consent forms were taken from each participant after providing a detailed oral explanation about the study project.

We retrospectively reviewed the medical files of all patients with the diagnosis of TBM admitted in 2014 to the CIIMS. From each medical case file, the patient's history, physical findings, chest radiographs, and the reports of laboratory investigations were assessed to obtain the necessary data about the diagnosis of TBM. For each patient, demographic information (age, gender), and clinical characteristics were also recorded.

The diagnosis of TBM was conducted according to internationally recognized clinical, radiological, and laboratory criteria (Torok, 2015). The clinical criteria included fever, headache, and neck stiffness.

The laboratory criteria included CSF pleocytosis of $>10$ cells per $\mathrm{mm} 3$ or proteins $>30 \mathrm{mg} / \mathrm{dL}$. The radiological criteria consisted of hydrocephalus, tuberculomas, cerebral infarcts, meningeal enhancements, or exudates. Additionally, the results of culture or staining of a clinical sample, Polymerase Chain Reaction (PCR) test, and Ag/ $\mathrm{Ab}$ test in the CSF, as well as tuberculin skin test were collected. A definite diagnosis of TBM was achieved when a positive culture or stain was present. A probable diagnosis was concluded when the clinical picture was compatible. A possible diagnosis was presented when there was a suggestive clinical picture and improvement with anti-TB treatment

In total, 278 cases were diagnosed with TBM in 2014 The cases with incomplete data or uncertain diagnosis were excluded from the study. Based on the abovementioned criteria, we separated 224 cases and followed them for 6 months and 12 months until 2015. These cases were clinically evaluated for new neurological complications, including the new formation or enlargement of cerebral lesions; the development of hydrocephalus; and the new onset of seizures or cranial nerve paralysis; the occurrence of intracranial hypertension; coma and death, or significant signs of improvement without new neurological deficits or symptoms.

Of the 224 TBM cases, 44 patients emigrated or were lost to follow-up within the first 6 months of diagnosis. Of the remaining 180 patients, $53.9 \%$ (97) of cases recovered completely, $36.1 \%$ (65) presented neurological deterioration, and $10 \%$ (18) died. 
Table 1. Primer sequences for RD region analysis

\begin{tabular}{|c|c|c|c|c|c|}
\hline PCR & Primers & Sequence & Annealing Temp & Amplicon Size & Reference \\
\hline RD4 & $\begin{array}{l}\mathrm{F} \\
\mathrm{R}\end{array}$ & $\begin{array}{l}\text { 5'-AATGGTTTGGTCATGACGCCTTC-3' } \\
\text { 5'-CCCGTAGCGTTACTGAGAAATTGC-3' }\end{array}$ & $58^{\circ} \mathrm{C}$ & $176 \mathrm{bp}$ & $\begin{array}{l}\text { Taylor } \\
\text { et al. (2007) }\end{array}$ \\
\hline RD1 & $\mathrm{F}$ & $\begin{array}{l}\text { 5'-CCCTTTCTCGTGTTTATAGTTTGA-3' } \\
\text { 5'-GCCATATCGTCCGGAGCTT-3' }\end{array}$ & $60^{\circ} \mathrm{C}$ & $110 \mathrm{bp}$ & $\begin{array}{l}\text { Halse, Escuyer, \& } \\
\text { Musser, (2011) }\end{array}$ \\
\hline
\end{tabular}

Table 2. The classification of study participants

\begin{tabular}{cc}
\hline Category * & No. of Cases \\
\hline Definite TBM & 24 \\
\hline Probable TBM & 123 \\
\hline Possible TBM & 77 \\
\hline Non-TBM & 34 \\
\hline
\end{tabular}

* Classification was performed on the basis of clinical, radiological, and CSF findings

NEUR SCIENCE

In a $12-$ month follow-up, these rates changed to $82.3 \%$ (84), 15.7\% (16), and 2\% (2) of recovered, deteriorated, and expired cases, respectively. The study workflow is represented in Figure 1. Along with the TBM group, a control group was included in the study, consisting of 34 non-TBM patients with viral (15) and fungal (4) meningitis, and patients with non-infectious illnesses of the CNS (15).

The potential for detecting mixed/new pathogenic infections in patients with poor prognosis led to the formulation of a novel strategy for differential diagnosis against the pathogenic mycobacteria. For this purpose, the Cerebrospinal Fluid (CSF) samples of TBM and non-TBM patients were processed for accurate identification of mycobacterial species; we used a duplex PCR assay targeting the Regions of Difference (RD) 1 and 4 in a single reaction.

CSF samples were collected under aseptic conditions by standard lumbar puncture. Five hundred microliter to $1 \mathrm{~mL}$ of sample was available for the study. The collected samples were stored at $-20^{\circ} \mathrm{C}$, prior to processing for target DNA for duplex PCR and culturing in the BACT alert system.

DNA was extracted from CSF samples by modifying the phenol-chloroform extraction method described by Deshpande et al. (2007); wherein $500 \mu \mathrm{L}$ of the sample was centrifuged at $12000 \mathrm{rpm}$ for $10 \mathrm{~min}$. The supernatant was discarded and the pellet suspended in $500 \mu \mathrm{L}$ of PBS, $15 \mu \mathrm{L}$
$10 \% \mathrm{SDS}$, and $3 \mu \mathrm{L}$ proteinase $\mathrm{K}(20 \mathrm{mg} / \mathrm{mL})$, mixed and incubated at $55^{\circ} \mathrm{C}$ for 1.5 hours. After incubation, $100 \mu \mathrm{L}$ of $5 \mathrm{M} \mathrm{NaCl}$ and $80 \mu \mathrm{L}$ of high-salt CTAB buffer (containing 4 $\mathrm{M} \mathrm{NaCl}, 1.8 \%$ CTAB (cetyl-trimethyl-ammonium bromide) was added and mixed; this process was followed by incubation at $65^{\circ} \mathrm{C}$ for $10 \mathrm{~min}$. An approximate equal volume (350 $\mu \mathrm{L})$ of phenol and chloroform-isoamyl alcohol (24:1) was added. Then, it was mixed thoroughly and centrifuged for 10 min in a microcentrifuge at 12000 RPM. The aqueous viscous supernatant was carefully decanted and transferred to a new tube. An equal volume of phenol: chloroform-isoamyl alcohol (1:1) was added followed by a $10 \mathrm{~min}$ spin at 12,000 RPM. The aqueous layer was separated. It was then mixed with $30 \mu \mathrm{L}$ of $3 \mathrm{M}$ sodium acetate and 0.6 volume of isopropanol to get a precipitate. The precipitated nucleic acids were washed with $70 \%$ ethanol, dried, and re-suspended in $30 \mu \mathrm{L}$ of Tris-EDTA (TE) buffer and stored at $-20^{\circ} \mathrm{C}$ before use. The DNA concentrations for all samples and strains used in this study were determined with the Quant-iTdsDNA HS assay kit using a Qubit fluorometer (Invitrogen).

For determining the species level of the mycobacterial pathogens, namely, Mycobacterium tuberculosis (M.tb), Mycobacterium Bovis (M. Bovis), and M. Bovis Bacilli Calmette Guérin (BCG), two genetic regions RD4 and RD1 were amplified using a duplex approach. Primers used in this study are presented in Table 1. 
Table 3. The clinical characteristics of the recruited participants

\begin{tabular}{|c|c|c|c|c|}
\hline \multirow{2}{*}{\multicolumn{2}{|c|}{ Characteristics }} & \multicolumn{2}{|c|}{ No. (\%)/ Mean士SD } & \multirow{2}{*}{$\mathbf{P}$} \\
\hline & & TBM Cases $(n=224)$ & Non-TBM Cases $(n=34)$ & \\
\hline & Male & $134(59.82)$ & $30(88.24)$ & 0.0045 \\
\hline \multirow[t]{2}{*}{ Gender } & Female & $90(40.18)$ & $4(11.76)$ & 0.0045 \\
\hline & Median age (y) & $37.19 \pm 17.67$ & $37.01 \pm 16.51$ & 0.9584 \\
\hline \multirow{8}{*}{ Clinical data } & Headache & 1655 (73.5) & $13(38.2)$ & 0.0004 \\
\hline & Fever & $134(59.8)$ & $10(29.4)$ & 0.0040 \\
\hline & Seizure & $46(20.5)$ & $3(8.8)$ & 0.1925 \\
\hline & Altered consciousness & $26(11.6)$ & $3(8.8)$ & 0.8671 \\
\hline & Altered sensorium & $99(44.2)$ & $6(17.6)$ & 0.0104 \\
\hline & Hallucinations & $6(2.7)$ & $2(5.9)$ & 0.7796 \\
\hline & Neck stiffness & $123(54.9)$ & $4(11.8)$ & $<0.0001$ \\
\hline & The duration of hospital stay & 30 & 15 & \\
\hline \multirow{2}{*}{ Radiological findings } & Hydrocephalus & $22(9.8)$ & $2(5.9)$ & 0.7299 \\
\hline & Meningeal enhancement & $70(31.3)$ & $6(17.6)$ & 0.1828 \\
\hline \multirow{3}{*}{ Laboratory results } & Proteins & 128.97 & 91.84 & 0.0322 \\
\hline & Glucose & 31.93 & 60.38 & $<0.0001$ \\
\hline & Cells & 209.72 & 83.75 & 0.0003 \\
\hline
\end{tabular}

RD4 is a region of difference in the bovine lineage. Employing RD4 flanking primers ensured that the PCR products were formed only if the deletion was present (Taylor, Worth, Palmer, Jahans, \& Hewinson, 2007). The genes of the $\mathrm{RD} 1$ region belong to the esat 6 gene cluster. ESAT-6 is a potent stimulator of the immune system, i.e. an antigen recognized during the early stages of infection. The RD1 region of M.tb is considered to be the primary attenuating deletion in the related vaccine strain M. bovis BCG (Halse, Escuyer, \& Musser, 2011) (Figure 2).

The duplex PCR reactions were conducted using 10X PCR buffer, $1.5 \mathrm{MgCl} 2,0.8 \mathrm{mM}$ dNTPs, $0.4 \mu \mathrm{M}$ of RD1F/R and $0.2 \mu \mathrm{M}$ of $\mathrm{RD} 4 \mathrm{~F} / \mathrm{R}$, and $1.25 \mathrm{U}$ of Taq DNA polymerase. The amplification procedure consisted of initial denaturation at $95^{\circ} \mathrm{C}$ for $7 \mathrm{~min}$ and 35 cycles each of denaturation at $95^{\circ} \mathrm{C}$ for $1 \mathrm{~min}$, annealing at $59^{\circ} \mathrm{C}$ for $1 \mathrm{~min}$, and extension at $72^{\circ} \mathrm{C}$ for $1 \mathrm{~min}$, followed by a final extension step at $72^{\circ} \mathrm{C}$ for $10 \mathrm{~min}$.

The sensitivity of the method was determined using serially diluted purified genomic DNA solutions-tenfold dilu- tion from $10 \mathrm{ng} / \mu \mathrm{L}$ to $1 \mathrm{FG} / \mu \mathrm{L}$; it was extracted from M.tb (ATCC 25177), M. Bovis (ATCC BAA-935), and M. Bovis BCG Pasteur (ATCC 35734). For the specificity study, the concentration of the DNA solution from each reference strain was adjusted to10 $\mathrm{ng} / \mu \mathrm{L}$, and used, accordingly.

The PCR amplicons were analyzed on a $2 \%$ agarose gel and stained with ethidium bromide. The amplified products were then visualized under UV light. Comparative analysis of electrophoresis of the PCR products generated by the two sets of primer pairs demonstrated the ability to distinguish between M.tuberculosis, M. bovis, and M. bovis BCG. The duplex PCR was considered as positive for M. bovis when the bands of 176-bp and 110-bp were detected; positive for M. Bovis BCG when the band of only 176-bp was present and positive for $\mathrm{M}$. TB when the band of only 110-bp was present.

The PCR products were purified and sequenced by Sanger's dideoxy chain termination method at the SciGenom Labs, Cochin, India. Sequences were verified by 


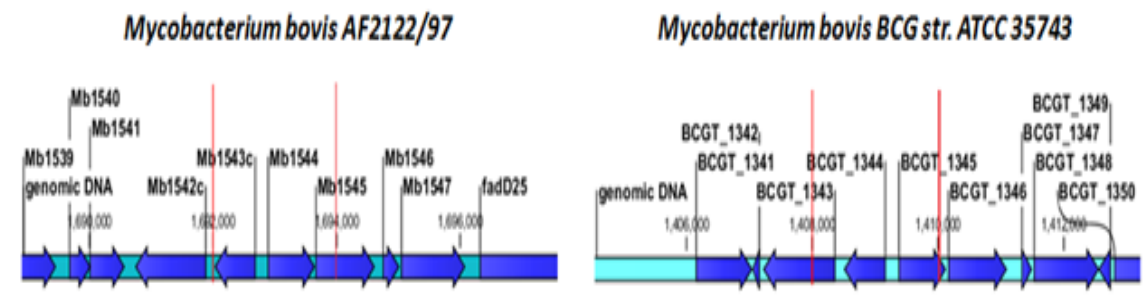

RD4 region of Mbovis and Mbovis BCG genomes giving 176-bp amplification product.

Mycobacterium bovis AF2122/97

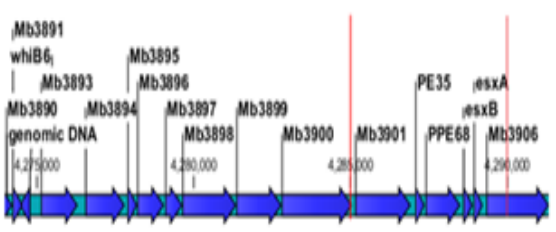

Mycobacterium tuberculosis CP009427

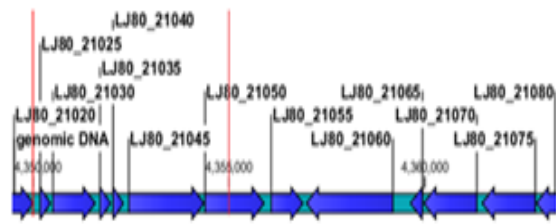

RD1 region of Mbovis and Mtuberculosis genomes giving 110-bp 176-bp amplification product.

NEUR SCIENCE

Figure 2. Molecular analysis for the identification and differentiation of Mycobacterium bovis (M. bovis), Mycobacterium bovisBCG (M. bovisBCG), and Mycobacterium tuberculosis (M.tb) using duplex PCR

* The position of the primers in the genomes is depicted.

BLAST search using the NCBI website. The positive culture isolates were subjected to antibiotic sensitivity using the 5 first-line drugs viz; Streptomycin, Isoniazid, Rifampicin, Ethambutol, and Pyrazinamide (SIREP) at Metropolis Healthcare Limited, Mumbai. In-vitro drug susceptibility testing was performed by incorporating required drug concentration and the subsequent inoculation of modified Middlebrook $7 \mathrm{H} 9$ Broth with standardized inoculum and incubated at $35^{\circ} \mathrm{C}$ in the automated BACTEC MGIT 960 system. Strains were declared resistant if the growth of $>20$ colonies was observed at drug concentrations as described by Rai, Bhattacharya, and Kamal (2007).

The recorded demographic and clinical characteristics were compared between TBM and non-TBM groups. The Chi-squared test for categorical variables was used to test the differences between the groups. Besides, $\mathrm{P} \leq$ 0.05 was considered as statistically significant. All tests were performed using MedCalc statistical software.

\section{Results}

Based on the clinical, radiological, and CSF findings, the recruited participants were categorized into definitive TBM $(n=24)$, probable TBM $(n=123)$, possible $\operatorname{TBM}(\mathrm{n}=77)$, and non-TBM cases $(\mathrm{n}=34)$ (Table 2$)$.

The overall male to female ratio of recruited cases was $1.7(164 / 94)$. For the TBM patients, the male to female ratio was $1.5(134 / 90)$, and 7.5 (30/4) for the non-TBM cases. The relevant difference was statistically significant $(\mathrm{P}<0.05)$. The median age of the TBM patients $(37.19$ years) and that of the non-TBM cases (37.01 years) was nearly similar (Table 3 ). A significantly higher proportion of the TBM cases experienced headache, fever, altered sensorium, and neck stiffness, compared to the non-TBM cases $(\mathrm{P}<0.01)$. On the other hand, a higher proportion of the non-TBM cases encountered hallucinations, compared to the TBM ones. A higher proportion of TBM cases had seizures and altered consciousness, compared to the non-TBM cases; however, such a difference was not statistically significant. The mean duration of illness was also significantly higher for TBM cases, compared to the non-TBM ones $(\mathrm{P}<0.0001)$. The frequency of cases that presented hydrocephalus and meningeal enhancement was also higher in the TBM cases, compared to the non-TBM cases. Laboratory findings indicated that the mean levels of protein and cell count in the TBM cases were significantly higher than those in the non-TBM cases $(\mathrm{P}<0.05)$. The glucose levels, however, were significantly lower in the non-TBM cases, compared to the TBM cases $(\mathrm{P}<0.0001)$.

Out of the total 224 TBM cases, 180 and 102 patients were followed up for 6 and 12 months, respectively. The radiological findings at the 6-month follow-up of the cases indicated that $22.8 \%$ had hydrocephalus, $3.9 \%$ had meningeal enhancements, and 1 case encountered stroke. 
Table 4. The follow-up clinical and radiological findings of the TBM cases

\begin{tabular}{|c|c|c|c|}
\hline & \multirow{2}{*}{ Characteristics } & \multicolumn{2}{|c|}{ No. (\%) } \\
\hline & & Follow-up* (6 M onths) & Follow-up" (12 Months) \\
\hline \multirow{3}{*}{ Clinical Findings } & Neurological Sequelae/Deterioration & $65(36.1)$ & $16(15.7)$ \\
\hline & Death & $18(10)$ & $2(2)$ \\
\hline & Improvement & $97(53.9)$ & $84(82.3)$ \\
\hline \multirow{3}{*}{ Radiological findings } & Hydrocephalus & $41(22.8)$ & $5(4.9)$ \\
\hline & Meningeal enhancement & $7(3.9)$ & $13(12.7)$ \\
\hline & Stroke & $1(0.6)$ & (0) \\
\hline \multirow{3}{*}{ Laboratory findings } & Proteins $>100 \mathrm{mg}$ & $48^{\natural}(26.7)$ & $18^{\text {" }}(17.6)$ \\
\hline & Glucose $<2 / 3$ CBS & $23^{\pi}(12.8)$ & $15^{\pi}(14.7)$ \\
\hline & Cells $>20$ cells $/ \mathrm{mm} 3$ & $34^{\uparrow}(18.9)$ & 13" (12.7) \\
\hline
\end{tabular}

NEUR SCIENCE

* $\mathrm{n}$ : 180 (44 cases lost to follow-up); ${ }^{n}$ : 102 (18 cases expired, lost to follow-up); " CSF was collected from patients with neurological deterioration.

In the second follow-up, $4.9 \%$ of the cases presented hydrocephalus, and $12.7 \%$ had meningeal enhancement. Protein, glucose, and cell count were found to be abnormal in $26.7 \%, 12.8 \%$, and $18.9 \%$ of the studied cases at the 6-month follow-up. At the 12-month follow-up step, these rates changed to $17.6 \%, 14.7 \%$, and $12.7 \%$ for protein, glucose, and total cell count, respectively. Table 4 indicates the follow-up clinical and radiological findings of the TBM cases.

The detection and differentiation of M.tb and M .bovis in CSF samples are represented in Figure 3. The PCR products in these samples were found to align with the
RD4 and RD1 regions of M. bovis (Figures 4A \& 4B). Of the 258 samples, $103(39.9 \%)$ were positive by the duplex PCR assay. M. bovis was detected in 7\% (18) of the investigated samples. Infection with M.tb was detected in $32.9 \%$ (85) of the samples. Furthermore, 14\% of the explored samples were positive for Bactec culture, M.tb or M. bovis; however, they could not be differentiated to the species level of the mycobacterial pathogens. The comparative efficiency of duplex PCR and Bactec culture for detecting mycobacteria are listed in Table 5 .

In-vitro drug susceptibility testing to first-line antitubercular drugs (SIREP) was outperformed and the sus-

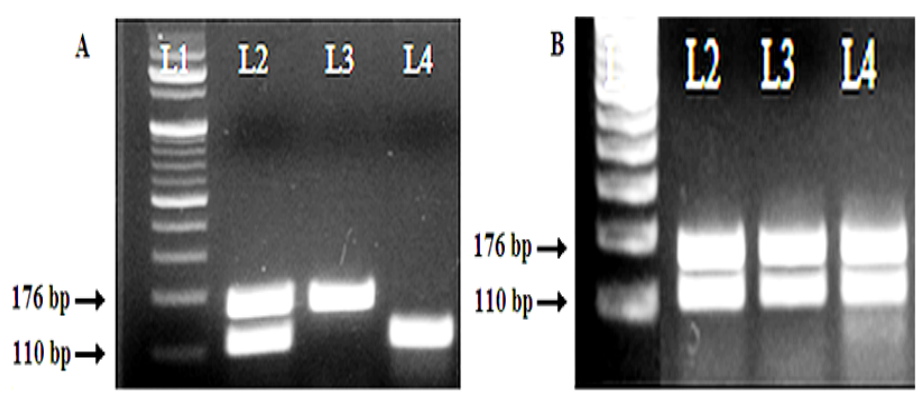

NEUROSCIENCE

Figure 3. Duplex PCR for detecting and differentiating M. bovis, M.tuberculosis, and M. bovis BCG.

A: The ethidium bromide-stained amplification products of L1: M. bovis, L2: M. bovisBCG and L3: M. tuberculosis when electrophoresced on $2 \%$ agarose gel. The $176 \mathrm{bp}$ and $110 \mathrm{bp}$ products obtained are indicated. B: L1: $100 \mathrm{bp}$ molecular ladder, L2: Positive control, L3 and L4: CSF samples with M. bovis infection. 


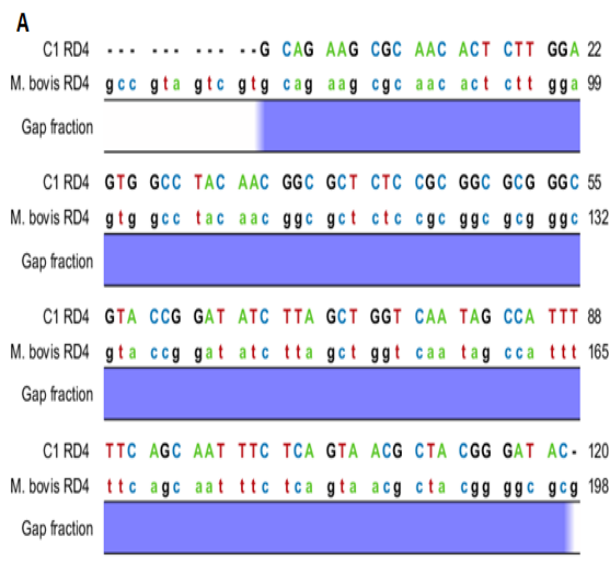

B

C1RD1 . G ACT ACG ACA AGC TCT TCC GGC 22

M. bovisRD1 ccg act acg aca agc tct tcc ggc 120

Consensus CCG ACT ACG ACA AGC TCT TCC GGC

Conservation

C1RD1 CGC ACG AAG GTA TGG AAG CTC CGG 46

M. bovisRD1 cgc acg aag gta $\operatorname{tgg}$ aag ctc cgg 144

Consensus CGC ACG AAG GTA TGG AAG CTC CGG

Conservation

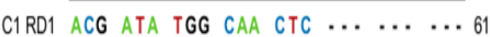

M. bovisRD1 acg ata $\operatorname{tgg} \mathrm{cag} \mathrm{cgc}$ agc cgt tct 168

Consensus ACG ATA TGG CAN CNC AGC CGT TCT

Conservation

NEUR SCIENCE

Figure 4. The follow-up clinical and radiological findings of the TBM cases

A: The alignment of M. bovis RD4 region and the PCR product of duplex PCR targeting the RD4 region in clinical sample from TBM patient (C1);

B. The alignment of M. bovis RD1 region and the PCR product of duplex PCR targeting the RD1 region in the same sample (C1)

*Sequence alignment was conducted using CLC Sequence Viewer, Version 6.6.1.

ceptibility pattern is detailed in Table 6 . One of the culture isolates was found to be resistant to pyrazinamide at a critical concentration of $100 \mu \mathrm{g} / \mathrm{mL}$. No other isolate reflected resistance to the first-line antitubercular drugs.

\section{Discussion}

The diagnosis of TBM has remained a challenge due to the low-efficiency and prolonged-time required by culture methods and conventional biochemical techniques. The paucibacillary nature of CSF has also been an additional impediment in the accurate diagnosis of the disease (Rock, Olin, Baker, Molitor, \& Peterson; Marx \& Chan, 2011).

We initiated this study to understand the variety of the presentations of TBM, including fatal and irrevocable effects occurring in a significant number of cases. We selected those patients diagnosed with TBM and followed them for one year to assess their neurological condition. With an underlying objective to clinically evaluate TBM patients with poor prognosis, we developed an assay based on the molecular detection of RD regions to accurately identify the causative organism in the CSF samples of these patients.

To our surprise, we found that $7 \%(18 / 258)$ of the cases diagnosed with TBM were infected with M. bovis, the classical causative agent of bovine TB; also reportedly responsible for human TB, which makes this bacterium an important zoonotic pathogen (Allix-Béguec, et al., 2010). In developed countries, the introduction of pasteurization and eradication programs for infected herds have considerably reduced the prevalence of human disease due to the bovine TB bacillus; however, have not completely eradicated it (Evans, et al., 2007). In developing countries, however, $\mathrm{M}$. bovis has been reported to account for approximately $10 \%$ to $15 \%$ of new human TB cases (Ashford, Whitney, \& Raghunathan, \& Cosivi, 2001).

The classical biochemical tests for identifying M. bovis and molecular methods based on targets, such as IS6110, 16S rDNA, 23S rDNA, or ITS cannot distinguish between $\mathrm{M}$. bovis and the other M.tbcomplex (MTBC) members. Other genetic markers and the single commercial test (GenoType Mycobacterium, Hain, Nehren, Germany) allowing the distinction between MTBC members, are not widely used due to the associated high costs (Sansila, et al., 1998). Diagnostically, the low sensitivity of CSF TB PCR is also problematic. Potential explanations for the lack of sensitivity in CSF specimens include low bacillary load in CSF, small sample volumes, and PCR inhibitors in the samples (Christie, et al., 2008). Moreover, from the clinicians' viewpoint, TB caused by M.tb. in humans, is clinically and radiologically identical to TB, caused by M. bovis (Grange, 2001). The distinction of M. bovis from M.tb however, has significant relevance to patient management. This is because M. bovis is intrinsically resistant to pyrazinamide; the absence of specific identification may have adverse consequences for infected cases (Niemann, Richter, \& Rüsch- Gerdes, 
Table 5. The comparative efficiency of duplex PCR and Bactec culture for the detection of mycobacteria

\begin{tabular}{cccccc}
\hline & \multicolumn{3}{c}{ No.(\%) } \\
\cline { 2 - 5 } Duplex PCR Result & \multirow{2}{c}{ Species Identified } & \multicolumn{2}{c}{ Culture Result } \\
\cline { 3 - 6 } & PCR Result & M.tuberculosis & M. bovis & Positive & Negative \\
\cline { 3 - 6 } & & $85(32.9)$ & $18(7.0)$ & $28(27.2)$ & $75(72.8)$ \\
Positive & $103(39.9)$ & $173(67.1)$ & $240(93.0)$ & $8(5.2)$ & $147(94.8)$ \\
Negative & $155(60.1)$ & $258(100)$ & $258(100)$ & $36(14)$ & $222(86)$ \\
\hline Total & $258(100)$ & & & & NEUR:SCIENCE
\end{tabular}

Table 6. The drug susceptibility testing of positive culture isolate

\begin{tabular}{ccc}
\hline Antitubercular Drug & Critical Concentration $(\mu \mathrm{g} / \mathrm{mL})$ & Interpretation \\
\hline Streptomycin & 1 & Susceptible \\
Isoniazid & 0.1 & Susceptible \\
Rifampicin & 1 & Susceptible \\
Ethambutol & 5 & Susceptible \\
Pyrazinamide & 100 & Resistant \\
\hline
\end{tabular}

2000). Identifying this pathogen would thus help clinicians to adopt desirable patient management concerning the treatment regimen. Reports by Allix-Béguec, et al. (2010) and Hannan, et al. (2001) have highlighted the significance of the routine use of molecular tests for the differentiation of M. bovis from M.tb or the systematic checking of resistance to pyrazinamide.

Various studies reported the presence of M. bovis infection in humans (Kidane, et al, 2002; Wei, Huang, Chu, Lee, 1999; LoBue, Betacourt, \& Peter, 2003; Cosivi, et al., 1998). In the Indian context, a study by Jain (2011) demonstrated M. bovis infection in $9.52 \%$ of the cases and the co-infection of M.tb and M. bovis in $4.76 \%$ of the cases through a two-step PCR, targeting the hup B gene. Prasad et al. (2005) reported that 34.7\% PCR positivity, of which M.tb was detected in $15.7 \%$ of the cases, M. bovis was observed in $10.3 \%$, and mixed infection in $8.7 \%$ of the samples. Shah et al. (2006) described applying a nested PCR (N-PCR) assay in detecting M.tb and M. bovis in human CSF. They reported the presence of M. bovis in $17 \%$ of the cases and mixed infection in $22 \%$ of them.

In the present study, of the total 258 cases, we detected M. bovis in 18 (7\%) cases; of which, two suffered a fatal outcome. One case; a 24-year-old pregnant woman
(ANC 26 weeks), was admitted with the typical symptoms of TBM, including altered sensorium, intermittent fever, decreased appetite, vomiting, and severe headache. Her CT scan presented meningeal enhancement, chest X-ray indicated Koch's infiltrations and subsequently initiated anti-TB treatment. The patient delivered a healthy premature baby during the hospital course and was discharged, subsequently. However, after 6 months, she re-developed symptoms, including abnormal behavior, marked neck-stiffness, and drowsiness. The patient was readmitted and surgically treated. The patient had however expired before the 12-month follow-up. Regardless and more importantly, the initial absence of $\mathrm{M}$. bovis identification of this patient's isolate compromised the efficiency of her treatments and plausibly influenced the final fatal outcome.

The second case, a 68-year-old male, had a history of right-sided pyothorax (tubercular) and infarction in corona radiata. He was discharged against medical advice from a local hospital, and consequently, admitted to CIIMS. The patient, upon admission, initiated anti-TB treatment and was discharged on improvement. The patient presented neurological sequelae upon follow-up and was readmitted; however, he suffered septic shock and died of multi-organ failure. In the remaining M. bovis in- 
fected cases, also the standard treatment regimen proved to be ineffective. It has led to neurological deterioration with symptoms, such as slurred speech, decreased hearing, and difficulty in walking. The assessment of the demographic characteristics of these individuals indicated that all the patients belonged to rural settings, where agriculture was the main source of livelihood. All the subjects had occupational exposure to farm animals. Close physical contact with animals and the consumption of unpasteurized milk could be the potential source of $\mathrm{M}$. bovis transmission in these individuals. Michel, Muller, and Van Helden (2010) suggested that pastoralist and rural communities are at greatest risk for zoonotic TB; however, the lack of data for these populations prevents the confirmation of this assumption.

While several articles have described the epidemiology, clinical features, and transmission of $\mathrm{M}$. bovis disease in humans, little has been documented about its treatment (LoBue \& Moser, 2005). Beyond its use for specific M. bovis identification, this natural resistance is particularly important to consider. Pyrazinamide is usually given in the classical first-line TB treatment; it is an effective sterilizing drug that helps to shorten TB therapy due to its synergistic effect with rifampicin. Thus, in the case of $M$. bovis infection, pyrazinamide would be ineffective if implemented in a patient's anti-TB regimen. The duration of treatment thus tended to be longer for patients with $\mathrm{M}$. bovis infection; they were considered ineligible for a 6-month treatment regimen due to PZA resistance (LoBue \& Moser, 2005). LoBue et al. also revealed that the death rate was highest for $\mathrm{M}$. bovis patients (LoBue \& Moser, 2005). In the present study, given the high rates of $\mathrm{M}$. bovis in TBM patients, the surveillance of this zoonotic pathogen was obviated.

\section{Conclusion}

Our study findings demonstrated the prevalence of $\mathrm{M}$. bovis-induced TB in patients with poor therapeutic outcomes. Besides, our report constituted an example of the persistent significance of $\mathrm{M}$. bovis, as a zoonotic pathogen. Human TB due to M. bovis is underestimated. This is because of the frequent use of diagnostic techniques that do not specifically distinguish M. bovis from other members of the M. tuberculosis complex, and because susceptibility to pyrazinamide is not systematically tested. Finally, the data demonstrated that molecular-guided cooperation between human and veterinary health services could improve the detection of zoonoses in the future.

\section{Ethical Considerations}

\section{Compliance with ethical guidelines}

The study was approved by Institutional Ethics Committee of Central India Institute of Medical Sciences (CIIMS), Nagpur and is in accordance with the Code of Ethics of the World Medical Association (Declaration of Helsinki). Written consents were taken from each participant or their kin after detailed oral explanation about the study.

\section{Funding}

This work was supported by the Indian Council of Medical Research (ICMR), New Delhi, India (Grant No: Zon.15/11/2014-ECD-II).

\section{Authors' contributions}

All authors were equally contributed in preparing this article.

\section{Conflict of interest}

The authors declared no conflicts of interest.

\section{Acknowledgments}

The authors acknowledge Dr. Dewanand R. Kalorey, Dr. Nitin V. Kurkure, and Dr. Sandeep P. Chaudhari for their support. The authors would also like to thank Ms. Sonali Manke and Ms. Ankita Tiwari for their technical assistance.

\section{References}

Ahmadinejad, Z., Ziaee, V., Aghsaeifar, M., \& Reiskarami, S. R. (2003). The prognostic factors of tuberculous meningitis. International Journal of Infectious Diseases, 3(1), 1-7. [DOI:10.5580/3a0]

Allix-Béguec, C., Fauville-Dufaux, M., Stoffels, K., Ommeslag, D., Walravens, K., Saegerman, C., \& Supply, P. (2010). Importance of identifying Mycobacterium bovis as a causative agent of human tuberculosis. European Respiratory Journal, 35(3), 692-4 [DOI:10.1183/09031936.00137309] [PMID]

Ashford, D. A., Whitney, E., Raghunathan, P., \& Cosivi, O. (2001). Epidemiology of selected mycobacteria that infect humans and other animals. Revue scientifique et technique (International Office of Epizootics), 20(1), 325-37. [DOI:10.20506/rst.20.1.1266] [PMID]

Christie, L. J., Loeffler, A. M., Honarmand, S., Flood, J. M., Baxter, R., \& Jacobson, S., et al. (2008). Diagnostic challenges of central nervous system tuberculosis. Emerging Infectious Diseases, 14(9), 1473. [DOI:10.3201/eid1409.070264] [PMID] [PMCID] 
Cosivi, O., Grange, J. M., Daborn, C. J., Raviglione, M. C., Fujikura, T., \& Cousins, D., et al. (1998). Zoonotic tuberculosis due to Mycobacterium bovis in developing countries. Emerging Infectious Diseases, 4(1), 59-70. [DOI:10.3201/eid0401.980108] [PMID] [PMCID]

Deshpande, P. S., Kashyap, R. S., Ramteke, S. S., Nagdev, K. J., Purohit, H. J., Taori, G. M., \& Daginawala, H. F. (2007). Evaluation of the IS6110 PCR assay for the rapid diagnosis of tuberculous meningitis. Cerebrospinal Fluid Research, 4(1), 10. https:// link.springer.com/article/10.1186/1743-8454-4-10

Evans, J. T., Smith, E. G., Banerjee, A., Smith, R. M., Dale, J., \& Innes, J. A., et al. (2007). Cluster of human tuberculosis caused by Mycobacterium bovis: Evidence for person-to-person transmission in the UK. The Lancet, 369(9569), 1270-6. [DOI:10.1016/S01406736(07)60598-4]

World Health Organization. (2020). Global tuberculosis report 2015 - World Health Organization; Geneva: World Health Organization. https://www.who.int/emergencies/diseases/novel-

Grange, J. M. (2001). Mycobacterium bovis infection in human beings. Tuberculosis, 81(1-2), 71-7. [DOI:10.1054/tube.2000.0263] [PMID]

Halse, T. A., Escuyer, V. E., \& Musser, K. A. (2011). Evaluation of a single-tube multiplex real-time PCR for differentiation of members of the Mycobacterium tuberculosis complex in clinical specimens. Journal of Clinical Microbiology, 49(7), 2562-7. [DOI:10.1128/ JCM.00467-11] [PMID] [PMCID]

Hannan, M. M., Desmond, E. P., Morlock, G. P., Mazurek, G. H., \& Crawford, J. T. (2001). Pyrazinamide-monoresistant Mycobacterium tuberculosis in the United States. Journal of Clinical Microbiology, 39(2), 647-50. [DOI:10.1128/JCM.39.2.647-650.2001] [PMID] [PMCID]

Jain, A. (2011). Extra pulmonary tuberculosis: A diagnostic dilemma. Indian Journal of Clinical Biochemistry, 26(3), 269-73. [DOI:10.1007/s12291-010-0104-0] [PMID] [PMCID]

Kashyap, R. S., Dobos, K. M., Belisle, J. T., Purohit, H. J., Chandak, N. H., \& Taori, G. M., et al. (2005). Demonstration of components of antigen 85 complex in cerebrospinal fluid of tuberculous meningitis patients. Clinical and Diagnostic Laboratory Immunology, 12(6), 752-8. [DOI:10.1128/CDLI.12.6.752-758.2005] [PMID] [PMCID]

Kashyap, R. S., Kainthla, R. P., Mudaliar, A. V., Purohit, H. J., Taori, G. M., \& Daginawala, H. F. (2006). Cerebrospinal fluid adenosine deaminase activity: A complimentary tool in the early diagnosis of tuberculous meningitis. Cerebrospinal fluid research, 3(1), 5. https://link.springer.com/article/10.1186/1743-8454-3-5

Kaur, H., Sharma, K., Modi, M., Sharma, A., Rana, S., \& Khandelwal, N., et al. (2015). Prospective analysis of 55 cases of Tuberculosis Meningitis (TBM) in North India. Journal of Clinical and Diagnostic Research, 9(1), DC15. [DOI:10.7860/JCDR/2015/11456.5454] [PMID] [PMCID]

Kidane, D., Olobo, J. O., Habte, A., Negesse, Y., \& Aseffa, A., Abate, G., et al. (2002). Identification of the causative organism of tuberculous lymphadenitis in Ethiopia by PCR. Journal of Clinical Microbiology, 40(11), 4230-4. [DOI:10.1128/JCM.40.11.42304234.2002] [PMID] [PMCID]

LoBue, P. A., \& Moser, K. S. (2005). Treatment of mycobacterium bovis infected tuberculosis patients: San Diego County, Califor- nia, United States, 1994-2003. The International Journal of Tuberculosis and Lung Disease, 9(3), 333-8. https://www.ingentaconnect com/content/iuatld/iitld/2005/00000009/00000003/art00019

LoBue P, A., Betacourt, W., Peter, C., \& Moser K, S. (2003). Epidemiology of Mycobacterium bovis disease in San Diego County, 1994-2000. The International Journal of Tuberculosis and Lung Disease, 7(2), 180-5. https://www.ingentaconnect.com/ content/iuatld/ijtld/2003/00000007/00000002/art00013

Marx, G. E., \& Chan, E. D. (2011). Tuberculous meningitis: diagnosis and treatment overview. Tuberculosis Research and Treat ment, 2011. [DOI:10.1155/2011/798764] [PMID] [PMCID]

Michel, A. L., Müller, B., \& Van Helden, P. D. (2010). Mycobacterium bovis at the animal-human interface: A problem, or not? Veterinary Microbiology, 140(3-4), 371-381. [DOI:10.1016/j. vetmic.2009.08.029] [PMID]

Murthy, J. M. K. (2010). Tuberculous meningitis: The challenges. Neurology India, 58(5), 716. [DOI:10.4103/0028-3886.72178] [PMID]

Niemann, S., Richter, E., \& Rüsch-Gerdes, S. (2000). Differentiation among Members of the Mycobacterium tuberculosis Complex by Molecular and Biochemical Features: Evidence for Two Pyrazinamide-Susceptible Subtypes of M. bovis. Journal of Clinical Microbiology, 38(1), 152-7. https://jcm.asm. org/content/38/1/152.short

Prasad, H. K., Singhal, A., Mishra, A., Shah, N. P., Katoch, V. M., \& Thakral, S. S., et al. (2005). Bovine tuberculosis in India: potential basis for zoonosis. Tuberculosis, 85(5-6), 421-8. [DOI:10.1016/j.tube.2005.08.005] [PMID]

Purohit, M., and Mustafa, T. (2015). Laboratory Diagnosis of Extra-pulmonary Tuberculosis (EPTB) in Resource-constrained Setting: State of the Art, Challenges and the Need. Journal of Clinical and Diagnostic Research, 9(4), EE01-EE06. [DOI:10.7860/JCDR/2015/12422.5792] [PMID] [PMCID]

Rai, S. P., Bhattacharyya, D., \& Kashyap, M. (2007). Pattern of initial drug resistance and its impact on short course chemotherapy of pulmonary tuberculosis. Lung India, 24(2), 51-3. [DOI:10.4103/0970-2113.44210]

Rock, R. B., Olin, M., Baker, C. A., Molitor, T. W., \& Peterson, P. K. (2008). Central nervous system tuberculosis: pathogenesis and clinical aspects. Clinical Microbiology Reviews, 21(2), 243-61. [DOI:10.1128/CMR.00042-07] [PMID] [PMCID]

Sansila, A., Hongmanee, P., Chuchottaworn, C., Rienthong, S., Rienthong, D., \& Palittapongarnpim, P. (1998). Differentiation between Mycobacterium tuberculosis and Mycobacterium avium by Amplification of the 16S-23S Ribosomal DNA Spacer. Journal of Clinical Microbiology, 36(9), 2399-403. [DOI:10.1128/JCM.36.9.2399-2403.1998] [PMID] [PMCID]

Shah, N. P., Singhal, A., Jain, A., Kumar, P., Uppal, S. S., \& Srivatsava, M. V. P., et al. (2006). Occurrence of overlooked zoonotic tuberculosis: detection of Mycobacterium bovis in human cerebrospinal fluid. Journal of Clinical Microbiology, 44(4), 1352-8. [DOI:10.1128/JCM.44.4.1352-1358.2006] [PMID] [PMCID]

Taylor, G. M, Murphy, E., Hopkins, R., Rutland, P., Chistov, Y. (2007). First report of Mycobacterium bovis DNA in human remains from the Iron Age. Microbiology (Reading), 153(Pt 4), 1243-9. [DOI:10.1099/mic.0.2006/002154-0] [PMID] 
Taylor, G. M., Worth, D. R., Palmer, S., Jahans, K., \& Hewinson, R. G. (2007). Rapid detection of Mycobacterium bovis DNA in cattle lymph nodes with visible lesions using PCR. BMC Veterinary Research, 3(1), 1-11. [DOI:10.1186/1746-6148-3-12] [PMID] [PMCID]

Török, M. E. (2015). Tuberculous meningitis: Advances in diagnosis and treatment. British Medical Bulletin, 113(1), 117-31. [DOI:10.1093/bmb/ldv003] [PMID]

Wei, C. Y., Hwang, J. J., Chu, C. H., \& Lee, C. P. (1999). Detection and identification of Mycobacterium tuberculosis by nested PCR assays in cerebrospinal fluid samples from patients with suspected tuberculous meningitis. The Kaohsiung Journal of Medical Sciences, 15(8), 475-83. [DOI:10.6452/ KJMS.199908.0475] 